COMMUNICATIONS IN

ANALYSIS AND GEOMETRY

Volume 14, Number 1, 163-182, 2006

\title{
Mass endomorphism and spinorial Yamabe type problems on conformally flat manifolds
}

\author{
Bernd Ammann ${ }^{1}$, Emmanuel Humbert and Bertrand Morel
}

Let $M$ be a compact manifold equipped with a Riemannian metric $g$ and a spin structure $\sigma$. We let $\lambda_{\min }^{+}(M,[g], \sigma)=$ $\inf _{\tilde{g} \in[g]} \lambda_{1}^{+}(\tilde{g}) \operatorname{Vol}(M, \tilde{g})^{1 / n}$ where $\lambda_{1}^{+}(\tilde{g})$ is the smallest positive eigenvalue of the Dirac operator $D$ in the metric $\tilde{g}$. A previous result stated that $\lambda_{\min }^{+}(M,[g], \sigma) \leq \lambda_{\min }^{+}\left(\mathbb{S}^{n}\right)=\frac{n}{2} \omega_{n}^{1 / n}$ where $\omega_{n}$ stands for the volume of the standard $n$-sphere. In this paper, we study this problem for conformally flat manifolds of dimension $n \geq 2$ such that $D$ is invertible. E.g., we show that strict inequality holds in dimension $n \equiv 0,1,2 \bmod 4$ if a certain endomorphism does not vanish. Because of its tight relations to the ADM mass in General Relativity, the endomorphism will be called mass endomorphism. We apply the strict inequality to spin-conformal spectral theory and show that the smallest positive Dirac eigenvalue attains its infimum inside the enlarged volume-1-conformal class of $g$.

\section{Introduction.}

Let $(M, g, \sigma)$ be a compact spin manifold of dimension $n \geq 2$. For a metric $\tilde{g}$ in the conformal class $[g]$ of $g$, let $\lambda_{1}^{+}(\tilde{g})$ be the smallest positive eigenvalue of the Dirac operator $D$. Similarly, let $\lambda_{1}^{-}(\tilde{g})$ be the largest negative eigenvalue of $D$. We define

$$
\lambda_{\min }^{+}(M,[g], \sigma)=\inf _{\tilde{g} \in[g]} \lambda_{1}^{+}(\tilde{g}) \operatorname{Vol}(M, \tilde{g})^{1 / n}
$$

and

$$
\lambda_{\min }^{-}(M,[g], \sigma)=\inf _{\tilde{g} \in[g]}\left|\lambda_{1}^{-}(\tilde{g})\right| \operatorname{Vol}(M, \tilde{g})^{1 / n}
$$

It was proven in $[2]$ that

$$
\left.\lambda_{\min }^{+}(M,[g], \sigma)>0 \quad \text { and } \quad \lambda_{\min }^{-}(M,[g], \sigma)\right)>0 .
$$

\footnotetext{
${ }^{1}$ Ammann was supported by the NSF grant DMS-9810361 of MSRI, Berkeley.
} 
Several works have been devoted to the study of this conformal invariant. A non-exhaustive list is $[12,13,17,8,3,4]$. In [2], we proved the following result:

Theorem 1.1. Let $(M, g, \sigma)$ be a compact spin manifold of dimension $n \geq 2$. Then

$$
\lambda_{\min }^{+}(M,[g], \sigma) \leq \lambda_{\min }^{+}\left(\mathbb{S}^{n}\right)=\frac{n}{2} \omega_{n}^{\frac{1}{n}} \text { and } \lambda_{\min }^{-}(M,[g], \sigma) \leq \lambda_{\min }^{+}\left(\mathbb{S}^{n}\right),
$$

where $\omega_{n}$ stands for the volume of the standard sphere $\mathbb{S}^{n}$.

The strict inequalities

$$
\lambda_{\min }^{+}(M,[g], \sigma)<\frac{n}{2} \omega_{n}^{\frac{1}{n}} \text { and } \lambda_{\min }^{-}(M,[g], \sigma)<\frac{n}{2} \omega_{n}^{\frac{1}{n}}
$$

have several applications. At first, when $n \geq 2$, together with Hijazi inequality, each one of the two inequalities (1.1) implies the existence of a solution of the Yamabe problem. This problem is a famous problem of conformal geometry which has been solved by Aubin [7] and Schoen [18].

Another application of the inequalities (1.1) is the solution of a conformally invariant PDE which can be read as a non-linear eigenvalue equation for the Dirac operator. The non-linearity involves a critical exponent from the point of view of Sobolev embeddings and hence, this PDE cannot be solved by standard methods. Moreover, from this solution, one can construct a generalized metric $\tilde{g}$ (see $[4,3]$ ) such that $\operatorname{Vol}_{\tilde{g}}(M)=1$ and such that $\lambda_{1}^{+}(\tilde{g})=\lambda_{\min }^{+}(M,[g], \sigma)$ (resp. $\quad \lambda_{1}^{-}(\tilde{g})=\lambda_{\min }^{-}(M,[g], \sigma)$ ). In other words, this proves that $\lambda_{\min }^{+}(M,[g], \sigma)$ (resp. $\left.\lambda_{\min }^{-}(M,[g], \sigma)\right)$ is attained by the generalized metric $\tilde{g}$.

In this paper, we are interested in obtaining the inequalities (1.1) when $M$ is a conformally flat manifold of dimension $n \geq 2$ such that $D$ is invertible. In this goal, we introduce in Section 2 the notion of mass endomorphism. This endomorphism corresponds to the constant term in the development of the Green function for $D$ near the diagonal with respect to a conformal chart. In Remark 2.12, we will show that the pointwise eigenvalues of the mass endomorphism are all real. The mass endomorphism plays the same role as the constant term of the Green function $\gamma(\cdot, p)$ of the Yamabe operator. In the Yamabe problem, the constant term of $\gamma$ can be interpreted as the mass [1] of the asymptotically flat manifold $\left(M \backslash\{p\}, \gamma(\cdot, p)^{4 /(n-2)} g\right)$ (see also [16]). This is why we use the name mass endomorphism. Schoen shows in [18] that the positivity of the mass implies the solution of the Yamabe problem. In this paper, the eigenvalues of the mass endomorphism play the 
same role as the mass in Yamabe problem. Namely, we obtain the following result:

Theorem 1.2. Let $(M, g, \sigma)$ be a conformally flat compact spin manifold of dimension $n \geq 2$ with $\operatorname{ker}(D)=\{0\}$. Assume that the mass endomorphism (see next section) possesses a positive (resp. negative) eigenvalue. Then,

$$
\lambda_{\min }^{+}(M,[g], \sigma) \quad\left(\text { resp. } \lambda_{\min }^{-}(M,[g], \sigma)\right)<\lambda_{\min }^{+}\left(\mathbb{S}^{n}\right)=\frac{n}{2} \omega_{n}^{\frac{1}{n}} .
$$

Assume that $n \not \equiv 3 \bmod 4$, then the spectrum of the Dirac operator and the pointwise spectrum of the mass endomorphism are symmetric (see Subsection 2.5). In particular,

$$
\lambda_{\min }^{+}(M,[g], \sigma)=\lambda_{\min }^{-}(M,[g], \sigma) .
$$

This implies

Theorem 1.3. Let $(M, g, \sigma)$ be a conformally flat compact spin manifold of dimension $n \geq 2$ with $\operatorname{ker}(D)=\{0\}$. Assume that $n \not \equiv 3 \bmod 4$ and that the mass endomorphism $\alpha$ is not identically zero. Then,

$\lambda_{\min }^{+}(M,[g], \sigma)<\lambda_{\min }^{+}\left(\mathbb{S}^{n}\right)=\frac{n}{2} \omega_{n}^{\frac{1}{n}}$ and $\lambda_{\min }^{-}(M,[g], \sigma)<\lambda_{\min }^{+}\left(\mathbb{S}^{n}\right)=\frac{n}{2} \omega_{n}^{\frac{1}{n}}$.

This is no longer true if $n \equiv 3 \bmod 4$. In Example 2.15, we study the real projective spaces $\mathbb{R} P^{4 k+3}$. Here, the mass endomorphism is a non-vanishing multiple of the identity section, hence has constant sign which depends on the spin structure. Furthermore, the two spin structures $\sigma_{+}$and $\sigma_{-}$on $\mathbb{R} P^{4 k+3}$ satisfy

$$
\begin{aligned}
& \lambda_{\text {min }}^{-}\left(\mathbb{R} P^{4 k+3}, g_{0}, \sigma_{+}\right)>\lambda_{\text {min }}^{+}\left(\mathbb{R} P^{4 k+3}, g_{0}, \sigma_{+}\right)=\frac{n}{2}\left(\frac{\omega_{n}}{2}\right)^{\frac{1}{n}}, \\
& \lambda_{\text {min }}^{+}\left(\mathbb{R} P^{4 k+3}, g_{0}, \sigma_{-}\right)>\lambda_{\text {min }}^{-}\left(\mathbb{R} P^{4 k+3}, g_{0}, \sigma_{-}\right)=\frac{n}{2}\left(\frac{\omega_{n}}{2}\right)^{\frac{1}{n}} .
\end{aligned}
$$

Hence, as predicted by Theorem 1.2 either $\lambda_{\min }^{+}$or $\lambda_{\text {min }}^{-}$is smaller than $(n / 2) \omega_{n}^{1 / n}$. As a remark, if $\min \left(\lambda_{\min }^{+}\left(\mathbb{R} P^{4 k+3}, g_{0}, \sigma_{-}\right), \lambda_{\min }^{-}\left(\mathbb{R} P^{4 k+3}, g_{0}, \sigma_{-}\right)\right)$ $<(n / 2) \omega_{n}^{1 / n}$, then the infimum in the definition of $\lambda_{\text {min }}^{ \pm}$would be attained by a metric of non-constant curvature. It is then natural to think that we cannot obtain the strict inequality (1.1) for $\lambda_{\min }^{+}$and $\lambda_{\text {min }}^{-}$for all manifolds of dimension $n \equiv 3 \bmod 4$. 
In order to prove inequalities (1.1) for arbitrary conformally flat manifolds of dimension $n \geq 2$ such that $D$ is invertible, then one has to find some non-zero eigenvalues of the mass endomorphism. Some questions arise naturally. At first, on the sphere $\mathbb{S}^{n}$, the mass endomorphism is null. Otherwise, we could apply Theorem 1.2. One may wonder if the mass endormorphism is not always zero. The answer is no since as mentioned above, the projective spaces give examples for which the mass endomorphism is a non-zero multiple of the identity.

Recall once again that the constant term of the Green function of the Yamabe operator can be interpreted as the mass of an asymptotically flat manifold; according to the positive mass theorem, it is positive unless $M$ is conformally diffeomorphic to $\mathbb{S}^{n}$. Then, one could hope to find a result of the same type for the mass endomorphism. However, we show that this is false in general. Namely, we show in Section 4 that the mass endomorphism of flat tori always vanishes.

\section{The mass endomorphism.}

In the following, we assume that $(M, g, \sigma)$ is a conformally flat manifold of dimension $n \geq 2$. We also assume that $D$ is invertible, i.e., that $\operatorname{ker}(D)=\{0\}$. The mass endomorphism is defined as the constant term in the Green function for $D$. In this section, we give a precise definition and some properties of the mass endomorphism.

\subsection{Trivialization of the spinor bundle.}

Let $\rho: U \subset(M, g) \rightarrow V \subset\left(N, g_{N}\right)$ be a conformal map preserving orientation and spin structure. We write $g=f^{2} \rho^{*} g_{N}$. Then, according to $[14,12]$, there is a fiberwise isomorphism

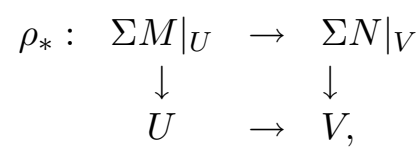

such that $D_{N} \rho_{*} \varphi=f \rho_{*} D \varphi$ for all $\varphi \in \Gamma(\Sigma M)$ and $f^{-\frac{n-1}{2}} \rho_{*}$ is an isometry, where $D$ and $D_{N}$ denote the Dirac operators on $M$ and $N$. The most important case we will use is that $N$ is Euclidean space, i.e. $\rho$ is a conformal chart of $(M, g)$ preserving orientation and the spin structure. In this case, the above map yields a well chosen trivialization of the spinor bundle.

The definition of the mass endomorphism will be done by working with a conformal chart. For simplicity, we will first define it in the special case 
that $g$ is flat in a neighborhood of a given base point, and then extend it to the general case.

\subsection{Green function for the Dirac operator.}

Let $\pi_{1}, \pi_{2}: M \times M \rightarrow M$ be the projection to the first and second component. Then, we define

$$
\Sigma M \otimes \Sigma M^{*}:=\pi_{1}^{*}(\Sigma M) \otimes\left(\pi_{2}^{*}(\Sigma M)\right)^{*},
$$

i.e., it is the bundle whose fiber over $(x, y)$ is given by $\operatorname{Hom}\left(\Sigma_{y} M, \Sigma_{x} M\right)$. Let $\Delta:=\{(p, p) \mid p \in M\}$ be the diagonal.

Definition 2.1. A smooth section $G_{D}: M \times M \backslash \Delta \rightarrow \Sigma M \otimes \Sigma M^{*}$ that is locally integrable on $M \times M$ is called the Green function for the Dirac operator $D$ if in the sense of distributions

$$
D_{x}\left(G_{D}(x, y)\right)=\delta_{y} I d_{\Sigma_{y} M}
$$

In other words, we have for any $y \in M, \psi_{0} \in \Sigma_{y} M$, and $\varphi \in \Gamma(\Sigma M)$

$$
\int\left\langle G_{D}(x, y) \psi_{0}, D^{*} \varphi(x)\right\rangle d x=\left\langle\psi_{0}, \varphi(y)\right\rangle
$$

For $k: M \times M \backslash \Delta \rightarrow \Sigma M \otimes \Sigma M^{*}$, we denote the corresponding integral operator by $P_{k}$, i.e. we define for $\varphi \in \Gamma(\Sigma M)$

$$
P_{k}(\varphi)(x):=\int k(x, y) \varphi(y) d y
$$

If $k$ is smooth on $M \times M \backslash \Delta$ and locally integrable on $M \times M$, then the integral exists in the principal value sense of distributions. $P_{k}$ uniquely determines $k$. In this notation, $P_{G_{D}}$ is the inverse of $D: L_{1}^{2}(\Sigma M) \rightarrow L^{2}(\Sigma M)$. Hence, the Green function is unique.

Remark 2.2. Analogously, one can define the Green function of Euclidean space as a section $G_{\text {eucl }}: \mathbb{R}^{n} \times \mathbb{R}^{n} \backslash \Delta \rightarrow \Sigma M \otimes \Sigma M^{*}$ such that $P_{G_{\text {eucl }}}$ is the inverse of $D_{\text {eucl }}: L_{1}^{2}(\Sigma M) \rightarrow L^{2}(\Sigma M)$. Again, we have unicity. One easily checks that

$$
G_{\text {eucl }}(x, y)=-\frac{1}{\omega_{n-1}} \frac{x-y}{|x-y|^{n}} .
$$


In our construction of the test spinor, we will need the asymptotics of Green functions close to the diagonal. This is provided by the following proposition.

Proposition 2.3. Assume that the metric is flat near $y \in M$, and let $\rho$ be an isometric chart. Then, the Green function $G_{D}$ for $D$ exists, and in the above trivialization has the following expansion when $x$ tends to $y$

$$
\omega_{n-1} G_{D}(x, y) \psi_{0}=-\frac{x-y}{|x-y|^{n}} \cdot \psi_{0}+v(x, y) \psi_{0}
$$

where $D_{x} v(x, y)\left(\psi_{0}\right)=0$ on a neighborhood of $y$.

Proof. Let $G_{D_{\text {eucl }}}$ be the Green function for $D_{\text {eucl }}$ on $\mathbb{R}^{n}$ given by Remark 2.2. We take a cut-off function $\eta$ with support in $B_{y}(\delta)$ which is equal to 1 on $B_{y}(\delta / 2)$ where $\delta>0$ is a small number. We set $\Phi(x)=\eta(x) G_{D_{\text {eucl }}}(x, y) \cdot \psi_{0}$ where $\psi_{0}$ is constant. The spinor $\Phi$ is harmonic on $B_{y}(\delta / 2) \backslash\{y\}$. We extend $\Phi$ by zero, and obtain a smooth spinor on $M \backslash\{y\}$. As $\left.D \Phi\right|_{B_{y}(\delta / 2)} \equiv 0$, we see that $D \Phi$ extends to a smooth spinor on $M$, denoted by $\Psi$. Since $D$ is assumed to be invertible, there exists a smooth spinor field $\zeta$ such that $D \zeta=-\Psi$. We then define $G_{D}(x, y) \psi_{0}:=\Phi(x)-\zeta(x)$. It is easy to see that $G_{D}$ is the Green function for $D$ and has the development described above. As $\Psi$ and hence $\zeta$ depend smoothly on $y$, it is clear that $G_{D}(x, y)$ depends smoothly on $y$ outside of the diagonal.

Lemma 2.4 (Conformal change and Green functions). Let $\rho$ : $\left(M, g_{M}, \sigma\right) \rightarrow\left(N, g_{N}, \sigma_{N}\right)$ be a conformal diffeomorphism preserving the orientation and the spin structure and write $g_{M}=f^{2} \rho^{*} g_{N}$. Let $G_{M}$ resp. $G_{N}$ be the Green function on $M$ resp. $N$, then

$$
G_{N}(\rho(x), \rho(y))=f^{n-1}(y) \rho_{*, x} \circ G_{M}(x, y) \circ \rho_{*, y}^{-1} .
$$

Proof. We already know that $D_{N}=f \rho_{*} D \rho_{*}^{-1}$. This implies

$$
P_{G_{N}}=D_{N}^{-1}=\rho_{*} D^{-1} \rho_{*}^{-1} f^{-1}=\rho_{*} P_{G_{M}} \rho_{*}^{-1} f^{-1},
$$

Hence,

$$
G_{N}(\rho(x), \rho(y)) d \mu_{N}(\rho(y))=f^{-1}(y) \rho_{*, x} \circ G_{M}(x, y) \circ \rho_{*, y}^{-1} d \mu(y) .
$$

With $d \mu=f^{n} d \mu_{N}$ this implies the lemma. 
In particular, the previous proposition and the lemma imply that the Green function $G_{D}$ for $D$ exists on any conformally flat manifold for which $D$ is invertible.

Example 2.5. If $\varphi_{\lambda}$ is an eigenspinor for the eigenvalue $\lambda$ of $D$, and if $G_{D}$ is the unique Green function for $D$, then

$$
\int_{M} G_{D}(x, y) \varphi_{\lambda}(y) d y=\frac{1}{\lambda} \varphi_{\lambda}(x)
$$

Example 2.6. Let $\rho: S^{n} \backslash\{N\} \rightarrow \mathbb{R}^{n}$ be the stereographic projection. Then

$$
g_{S^{n}}=\left(\frac{2}{|\rho(x)|^{2}+1}\right)^{2} \rho^{*} g_{\text {eucl }} .
$$

Let $G_{S^{n}}$ be the Green function on $S^{n}$. An obvious modification of Lemma 2.4 tells us that

$$
\rho_{*} G_{S^{n}}(x, y) \rho_{*}^{-1}=\left(\frac{2}{|\rho(y)|^{2}+1}\right)^{1-n} G_{\text {eucl }}(\rho(x), \rho(y)) .
$$

Example 2.7. Let $\rho: S^{n} \rightarrow S^{n}$ be a Moebius transformation of $S^{n}$ with $g_{S^{n}}=f^{2} \rho^{*}\left(g_{S^{n}}\right)$. Then

$$
G_{S^{n}}(x, y)=f^{n-1}(y) \rho_{*, x} \circ G_{S^{n}}(x, y) \circ \rho_{*, y}^{-1} .
$$

In the sequel, the following self-adjointness result for $G_{D}$ will be important.

Proposition 2.8. Let $V$ be an open set of $M$ in which $g$ is flat. Then, for all $x \neq y \in V, G_{D}(x, y)^{*}=G_{D}(y, x)$. In other words,

$$
\left\langle G_{D}(x, y) \psi_{y}, \varphi_{x}\right\rangle=\left\langle\psi_{y}, G_{D}(y, x) \varphi_{x}\right\rangle
$$

for all $\psi_{y} \in \Sigma_{y} M$ and $\varphi_{x} \in \Sigma_{x} M$.

Proof. We have

$$
P_{G_{D}}^{*}=\left(D^{-1}\right)^{*}=\left(D^{*}\right)^{-1}=D^{-1}=P_{G_{D}} .
$$

As the operator uniquely determines the kernel, this implies the proposition. 
Proposition 2.9. Assume that we have two metrics $g_{1}$ and $g_{2}$ on $M, g_{1}=$ $f^{2} g_{2}$. We assume that both metrics are flat in a neighborhood $U$ of $y$. We define $v_{1}$ and $v_{2}$ as above. Then

$$
v_{1}(x, x)=f^{1-n} v_{2}(x, x) .
$$

Proof. Let $G^{1}$ (resp. $G^{2}$ ) be the Green function for $g_{1}$ (resp. $g_{2}$ ). We identify spinors via $\rho_{*}$. By Lemma 2.4, $G^{1}$ and $G^{2}$ are related by the following formula: for all $x, y \in M$,

$$
G^{1}(x, y)=f^{1-n}(y) G^{2}(x, y)
$$

Let $h_{1}:\left(U, g_{1}\right) \rightarrow \mathbb{R}^{n}$ and $h_{2}:\left(U, g_{2}\right) \rightarrow \mathbb{R}^{n}$ be isometric embeddings. According to Liouville's theorem, the conformal map $h_{2} \circ h_{1}^{-1}: h_{1}(U) \rightarrow h_{2}(U)$ extends to a Möbius transformation of the Alexandrov compactification $S^{n}$ of $\mathbb{R}^{n}$. Because of Examples 2.6 and 2.7, this implies that

$$
G_{\text {eucl }}^{1}(x, y)=f^{1-n}(y) G_{\text {eucl }}^{2}(x, y) .
$$

Subtracting this from (2.1), and taking the limit $x \rightarrow y$, one obtains the desired formula.

\subsection{Definition and first properties of the mass endomorphism.}

The mass endomorphism is defined as the constant term of the Green function for $D$ with respect to a conformal chart. Let us make this precise.

Definition 2.10. Let $(M, g)$ be a compact manifold which is conformally flat on a neighborhood of $y \in M$. Choose a metric $\tilde{g} \in[g]$ that is flat on a neighborhood of $y$ and such that $\tilde{g}_{y}=g_{y}$. Let $G_{D}$ be the Green function for $D$. Then, we define the mass endomorphism as

$$
\alpha_{y}: \mid \begin{array}{ccc}
\Sigma_{y}(M) & \rightarrow & \Sigma_{y}(M) \\
\psi_{0} & \mapsto v(y, y)\left(\psi_{0}\right)
\end{array}
$$

where $v$ is as in the previous paragraph with respect to $\tilde{g}$.

Because of Proposition 2.9, this definition does not depend on the choice of $\tilde{g}$.

Proposition 2.11. For each $y \in M$, the mass endomorphism $\alpha_{y}$ is linear and self-adjoint. 
Proof. Let $y \in M$ and $\psi_{0}, \varphi_{0} \in \Sigma_{y}(M)$. We have

$$
\alpha_{y}\left(\psi_{0}\right)=\lim _{x \rightarrow y} \omega_{n-1} G_{D}(x, y) \psi_{0}+\frac{x-y}{|x-y|^{n}} \cdot \psi_{0}
$$

It follows immediately that $\alpha_{y}$ is linear. Taking the limit when $x \rightarrow y$, one gets that

$$
\left\langle\alpha_{y}\left(\psi_{0}\right), \varphi_{0}\right\rangle=\left\langle\psi_{0}, \alpha_{y}\left(\varphi_{0}\right)\right\rangle
$$

Remark 2.12. Proposition 2.11 immediately implies that the mass endomorphism has only real eigenvalues.

Remark 2.13. If $\operatorname{dim} M \equiv 2,3,4 \bmod 8$, then the spinor bundle carries a quaternionic structure, i.e. a basepoint-preserving, parallel, complex antilinear map $Q: \Sigma M \rightarrow \Sigma M$ with $Q^{-1}$ commuting with the Cliffordmultiplication. As a consequence, $Q$ commutes with $D, G_{D}$ and with $\alpha$.

\subsection{Examples.}

Example 2.14 (Flat tori). Let $(M, g)$ be an $n$-dimensional flat torus. It carries $2^{n}$ spin structures. For one spin structure, the so-called trivial spin structure, we have a $\operatorname{rank}(\Sigma M)=2^{[n / 2]}$-dimensional space of parallel sections. All other spin structures admit no non-trivial parallel spinors. Because $M$ is scalar-flat, the kernel of $D$ consists exactly of the parallel spinors, in particular $D$ is invertible for all non-trivial spin structures $\chi$. Because translations act spin-isometric on $(M, g, \chi)$, the Green function $G_{D}$ satisfies $G_{D}(x, y)=G_{D}(x-y, 0)$. Also $D_{x}\left(G_{D}(y, x)\right)=-\delta(x-y)$, hence $G_{D}(x, y)=-G_{D}(y, x)$. Therefore, all terms of even order in the development of $G_{D}$ have to vanish. In particular, the mass endomorphism vanishes.

Example 2.15 (Real Projective Spaces). Besides $\mathbb{R} P^{1}=S^{1}$, the only real projective spaces that are orientable and spin are $\mathbb{R} P^{4 k+3}$ with $k \in \mathbb{N}$. The space $M=\mathbb{R} P^{4 k+3}$ carries exactly two spin structures. The universal covering $\pi: S^{4 k+3} \rightarrow \mathbb{R} P^{4 k+3}$ induces a push-forward of the spinor bundles, which is a fiberwise isomorphism $\pi_{*}: \Sigma_{p} S^{4 k+3} \rightarrow \Sigma_{\pi(p)} \mathbb{R} P^{4 k+3}$. One calculates

$$
G_{D}^{\mathbb{R} P^{4 k+3}}(\pi x, \pi y) \circ \pi_{*}=\pi_{*} G_{D}^{S^{4 k+3}}(x, y)+\pi_{*} G_{D}^{S^{4 k+3}}(x,-y),
$$

where $-y$ denotes the antipodal point of $y$. Stereographic projection based in $y \notin\{x,-x\}$ defines a conformal chart containing $x$ and $-x$. Example 2.6 
implies that $G_{D}^{S^{4 k+3}}(x,-x) \neq 0$. Hence, the mass endomorphism $\alpha_{\pi(x)}$ of $\mathbb{R} P^{4 k+3}$ does not vanish anywhere on $\mathbb{R} P^{4 k+3}$.

The group of orientation preserving isometries fixing $\pi(x)$ is $\mathrm{SO}(4 k+3)$. After passing to the double cover $\operatorname{Spin}(4 k+3)$, we obtain a $\operatorname{Spin}(4 k+3)$ action on $\Sigma S^{4 k+3}$, that pushes down to a $\operatorname{Spin}(4 k+3)$-action on $\Sigma S^{4 k+3}$ which commutes with the Dirac operator. Hence, this action also commutes with the mass endomorphism, and as the $\operatorname{Spin}(4 k+3)$-action on $\Sigma_{\pi(x)} \mathbb{R} P^{4 k+3}$ is irreducible, the mass endomorphism is a constant multiple of the identity [15, Prop. I.5.15], [10, section 1.5]. If one changes the spin structure, then the second summand in (2.2) changes its sign. Hence, the sign of the mass endomorphism depends on the choice of the spin structure, which are denoted by $\sigma_{+}$and $\sigma_{-}$.

\subsection{Endomorphisms generating symmetries.}

The aim of this section is to show that if $n \not \equiv 3 \bmod 4$, then there is an automorphism $\Gamma\left(\operatorname{Aut}_{\mathbb{R}}(\Sigma M)\right)$ that anticommutes with the Dirac operator. This result is well-known, see for example [10, 1.7], [9, Prop. 5]. As a consequence, one sees that it also anticommutes with the mass endomorphism.

Let $(W, \gamma)$ be an irreducible complex representation of the Clifford algebra of an Euclidean vector space $V$ of dimension $n$. After fixing an orientation on $V$, one can define

$$
\omega_{\gamma}:=\gamma\left(e_{1} \cdot \ldots \cdot e_{n}\right) \in \operatorname{End}(W)
$$

where $e_{1}, \ldots, e_{n}$ is an oriented orthonormal basis on $V$, and where $\gamma$ denotes Clifford-multiplication. One easily calculates

$$
\omega^{2}:=(-1)^{n(n+1) / 2} \text {. }
$$

As a consequence, the eigenvalues of $\omega$ are contained in $\{-1,1\}$ if $n \equiv 0,3$ $\bmod 4$, and they are contained in $\{-i, i\}$ if $n \equiv 1,2 \bmod 4$.

2.5.1. The case $n \equiv 0 \bmod 2$. If $n$ is even, then Clifford-multiplication by a vector $v \in V$ anticommutes with $\omega$. Hence, if $\alpha$ is an eigenvalue $\omega$, then so is $-\alpha$. One immediately obtains the well-known lemma.

Lemma 2.16. If $n$ is even, then $\omega$ is a complex-linear automorphism of $W$ anticommuting with Clifford-multiplication.

Indeed, it can even be shown that up to automorphisms, there is only one irreducible representation in even dimensions $n$ and that any endomorphism anticommuting with Clifford-multiplication with vectors is a multiple of $\omega$. 
2.5.2. The case $n \equiv 1 \bmod 4$. The question arises, whether there is a similar endomorphism if $n$ is odd. In this case, Clifford-multiplication with a vector commutes with $\omega$. Hence, by Schur's lemma, $\omega$ has only one eigenvalue. For $n \equiv 1 \bmod 4$, we have either $\omega=i$ Id or $\omega=-i$ Id, and it can be shown, that there is exactly one irreducible representation of $V$ with $\omega=i$ Id denoted by $\left(W^{i}, \gamma^{i}\right)$, and one with $\omega=-i \mathrm{Id}$, denoted by $\left(W^{-i}, \gamma^{-i}\right)$. If we replace the complex structure on $W^{ \pm i}$, by its complex conjugate one, then this is again a representation of the (real) Clifford algebra of $V$. Obviously, $\omega$ changes sign by conjugation. Hence, there is a conjugate linear isomorphism of Clifford representation $\alpha: W^{i} \rightarrow W^{-i}$. Another way to modify the structure of $\left(W^{i}, \gamma^{i}\right)$ is to reverse the sign of Clifford-multiplication by vectors. Namely, we define a Clifford-multiplication $\tau^{i}: \mathbb{C} l(V) \rightarrow \operatorname{End}(W)$ as $\tau^{i}(X):=-\gamma^{i}(X)$ for all vectors $X$ in $V$. Again, we calculate that the sign of $\omega$ changes if we replace $\tau^{i}$ by $\gamma^{i}$, and there is a complex linear isomorphism of vector spaces $\beta: W^{-i} \rightarrow W^{i}$ with $\beta \circ \gamma^{-i}(X)=\tau^{i}(X) \circ \beta$ for any vector $X \in V$. Hence, $\nu:=\beta \circ \alpha: W^{i} \rightarrow W^{i}$ is a conjugate linear automorphism of vector spaces, and for vector $X$, we have

$$
\gamma^{i}(X) \circ \nu=-\tau^{i}(X) \circ \beta \circ \alpha=-\beta \circ \gamma^{i}(X) \circ \alpha=-\nu \circ \gamma^{i}(X) .
$$

A similar endomorphism of $W^{-i}$ is given by $\alpha \circ \beta$. We have proven the following lemma.

Lemma 2.17. If $n \equiv 1 \bmod 4$, then there is a real vector space automorphism of $W^{ \pm i}$ anticommuting with Clifford-multiplication by vectors. The automorphism is conjugate linear.

2.5.3. The case $n \equiv 3 \bmod 4 .$. The case $n \equiv 3 \bmod 4$ is different. Again, we have $\omega=\mathrm{Id}$ or $\omega=-\mathrm{Id}$, and there is exactly one irreducible representation $\left(W^{ \pm}, \gamma^{ \pm}\right)$in each case. However, conjugation does not exchange the representations, and the sign of $\omega$ is invariant under real automorphisms. Hence, an isomorphism as in the above lemma cannot exist.

2.5.4. For all $n \not \equiv 3 \bmod 4$.. Any automorphism $\nu$ anticommuting with Clifford-multiplication, commutes with bivectors $X \cdot Y$ where $X, Y \in V$. As the Lie algebra of $\operatorname{Spin}(n)$ is generated by elements of that form, such an isomorphisms $\nu$ is $\operatorname{Spin}(n)$-equivariant. We obtain the well-known

Proposition 2.18. If $n \equiv 0,1,2 \bmod 4$, then there is a real vector bundle isomorphism $\nu: \Sigma M \rightarrow \Sigma M$ anticommuting with Clifford-multiplication by 
vectors, complex linear if $n$ is even, and conjugate linear if $n \equiv 1 \bmod 4$. Furthermore, $\nu$ is parallel.

It follows that it anti-commutes with the Dirac operator, the Green function and the mass endomorphism.

Corollary 2.19 (Well-known, e.g. [6]). The spectrum of the Dirac operator is symmetric in dimension $n \not \equiv 3 \bmod 4$.

Corollary 2.20. The pointwise spectrum of the mass endomorphism is symmetric in dimension $n \not \equiv 3 \bmod 4$, i.e. if $\lambda$ is an eigenvalue of the mass endomorphism $\alpha_{x}$ for an $x \in M$, then $-\lambda$ is also an eigenvalue of $\alpha_{x}$ with the same multiplicity.

Corollary 2.21. If $\operatorname{dim} M=2$, then the mass endomorphism $\alpha$ vanishes.

Proof. The spectrum of $\alpha_{y}$ is symmetric and real. As $\alpha$ commutes with the quaternionic multiplication $Q$, the eigenspaces of $\alpha$ are quaternionic vector spaces. $\operatorname{dim} \Sigma_{y} M=2$ implies that $\alpha_{y}=r_{y} \mathrm{Id}$ for $r_{y} \in \mathbb{R}$. As the spectrum of $\alpha_{y}$ is symmetric, we obtain $r_{y}=0$.

\section{The estimates.}

Let $\psi \in \Gamma(\Sigma M)$ and define

$$
J(\psi)=\frac{\left(\int_{M}|D \psi|^{\frac{2 n}{n+1}} v_{g}\right)^{\frac{n+1}{n}}}{\int_{M} \Re e\langle D \psi, \psi\rangle v_{g}}
$$

The first named author proved in [2] that

$$
\lambda_{\min }^{+}(M,[g], \sigma)=\inf _{\psi} J(\psi)
$$

where the infimum is taken over the set of smooth spinor fields for which

$$
\left(\int_{M} \Re e\langle D \psi, \psi\rangle v_{g}\right)>0 \text {. }
$$

By adjusting some signs appropriately, one obtains by the same reasoning that

$$
\lambda_{\min }^{-}(M,[g], \sigma)=\inf _{\psi}-J(\psi)
$$


where the infimum is taken over the set of smooth spinor fields for which

$$
\left(\int_{M} \Re e\langle D \psi, \psi\rangle v_{g}\right)<0
$$

These two facts will be helpful to prove the following theorem

Theorem 3.1. Assume that there exists on $(M, g)$ a conformal metric $g_{p} \in$ $[g]$ which is flat in a neighborhood of a point $p$. If there exists on $M \backslash\{p\} a$ spinor field $\psi$ satisfying

- $D \psi=0$

- $\psi$ admits the following development near the point $p$ :

$$
\psi=\frac{x}{r^{n}} \cdot \psi_{0}+\psi_{1}+\theta
$$

where $\psi_{0}$ and $\psi_{1}$ are two spinors of $\Sigma_{p} M$ such that

$$
\Re e\left(\left\langle\psi_{0}, \psi_{1}\right\rangle\right)<0 \quad\left(\operatorname{resp} . \Re e\left(\left\langle\psi_{0}, \psi_{1}\right\rangle\right)>0\right),
$$

and where $\theta=O(r)$ is an harmonic spinor field smoothly defined in a neighborhood of $p$.

Then, we have

$$
\lambda_{\min }^{+}(M,[g], \sigma)<\frac{n}{2} \omega_{n}^{\frac{1}{n}} \quad\left(\text { resp. } \lambda_{\min }^{-}(M,[g], \sigma)<\frac{n}{2} \omega_{n}^{\frac{1}{n}}\right) .
$$

Proof. The proof is based on a suitable choice of a test spinor field $\psi_{\varepsilon}$ to estimate $J\left(\psi_{\varepsilon}\right)$. We let

$$
f(r):=\frac{1}{1+r^{2}} .
$$

As a first step, we consider for a given $\Phi$ the spinor defined by

$$
\varphi^{ \pm}(x):=f(|x|)^{\frac{n}{2}}(1 \mp x) \cdot \Phi \quad \forall x \in \mathbb{R}^{n} .
$$

One may compute that

$$
D\left(\varphi^{ \pm}\right)= \pm n f(|x|) \varphi^{ \pm} .
$$


Since the Euclidean space $\left(\mathbb{R}^{n} \backslash\{0\}, 4 f^{2} g_{\text {eucl }}\right)$ and the standard sphere $\left(\mathbb{S}^{n}, g_{0}\right)$ are isometric and using the conformal covariance of $D$, it is well known that there exists a natural map

$$
m \mid \begin{array}{ccc}
\Gamma\left(\Sigma\left(\mathbb{R}^{n} \backslash\{0\}\right)\right) & \rightarrow & \Gamma\left(\Sigma\left(\mathbb{S}^{n}\right)\right) \\
\varphi & \mapsto & m(\varphi)
\end{array}
$$

such that for all vector field $\varphi \in \Gamma\left(\Sigma\left(\mathbb{R}^{n} \backslash\{0\}\right)\right)$, we have

$$
m(D(\varphi))=2 f D_{\mathbb{S}^{n}}(m(\varphi))
$$

As one can check the spinor field $m\left(\varphi^{+}\right)$is a Killing spinor on $\mathbb{S}^{n}$ to the Killing constant $-\frac{1}{2}$, whereas $m\left(\varphi^{-}\right)$is a Killing spinor on $\mathbb{S}^{n}$ to the Killing constant $+\frac{1}{2}$. One gets that

$$
J_{\mathbb{R}^{n}}\left(\varphi^{ \pm}\right)= \pm \frac{n}{2} \omega_{n}^{\frac{1}{n}}
$$

where $J_{\mathbb{R}^{n}}$ is the functional $J$ written on $\mathbb{R}^{n}$. Now, fix $\varepsilon>0$ and let $\left(x_{1}, \cdots, x_{n}\right)$ be local coordinates on a neighborhood $U$ of $p$ in $M$. On $U$, we trivialize the spinor bundle via parallel transport. Using this trivialization, one may define for $x \in M$,

$$
\varphi_{\varepsilon}^{ \pm}:=\eta_{0} \varphi^{ \pm}\left(\frac{x}{\varepsilon}\right)
$$

where $\eta_{0}$ is a cut-off function equal to 1 on $B(p, \delta)$ ( $\delta$ is a small number).

We would like to have test spinors $\psi_{ \pm}$with $\pm \int\left\langle D \psi_{ \pm}, \psi_{ \pm}\right\rangle>0$ for which the strict inequalities

$$
\pm J\left(\psi_{ \pm}\right)<\frac{n}{2} \omega_{n}^{\frac{1}{n}}
$$

hold. For a given $\varepsilon>0$, we set

$$
\xi:=\varepsilon^{\frac{1}{n+1}} \quad \varepsilon_{0}:=\frac{\xi^{n}}{\varepsilon} f\left(\frac{\xi}{\varepsilon}\right)^{\frac{n}{2}} .
$$

The test spinor, we use here is the following:

$$
\psi_{\varepsilon}^{ \pm}:= \begin{cases}f\left(\frac{r}{\varepsilon}\right)^{\frac{n}{2}}\left(1 \mp \frac{x}{\varepsilon}\right) \cdot \psi_{0} \mp \varepsilon_{0} \psi_{1} & \text { if } r \leq \xi, \\ \mp \varepsilon_{0}(\psi-\eta \theta)+\eta f\left(\frac{\xi}{\varepsilon}\right)^{\frac{n}{2}} \psi_{0} & \text { if } \xi \leq r \leq 2 \xi, \\ \mp \varepsilon_{0} \psi & \text { if } r \geq 2 \xi,\end{cases}
$$


where $r=|x|$, where $\eta$ is a cut-off function which equals to 1 on $B(p, \xi)$, which is zero on the complement of $B(p, 2 \xi)$ and which statisfies

$$
|\nabla \eta| \leq \frac{2}{\xi}
$$

Note that $\psi_{\varepsilon}^{ \pm}$is continuous on $M$.

Remark 3.2. It should be pointed out that this choice of $\xi$ is arbitrary in the following sense. The proof of Theorem 3.1 still holds for any choice of $\xi=\varepsilon^{q}$ for $\left.q \in\right] \frac{n-1}{n(n+1)}, \frac{1}{n}[$.

We can assume without loss of generality that $\left|\psi_{0}\right|=1$. Since $\psi$ and $\theta$ are harmonic near $p$, we have

$$
D \psi_{\varepsilon}^{ \pm}= \begin{cases} \pm \frac{n}{\varepsilon} f\left(\frac{r}{\varepsilon}\right)^{\frac{n}{2}+1}\left(1 \mp \frac{x}{\varepsilon}\right) \cdot \psi_{0} & \text { if } r \leq \xi \\ \pm \varepsilon_{0} \nabla \eta \cdot \theta+f\left(\frac{\xi}{\varepsilon}\right)^{\frac{n}{2}} \nabla \eta \cdot \psi_{0} & \text { if } \xi \leq r \leq 2 \xi \\ 0 & \text { if } r \geq 2 \xi\end{cases}
$$

Therefore, since $\left|\left(1 \mp \frac{x}{\varepsilon}\right) \cdot \psi_{0}\right|^{2}=\left(1+\frac{r^{2}}{\varepsilon^{2}}\right)\left|\psi_{0}\right|^{2}=f\left(\frac{r}{\varepsilon}\right)^{-1}$, we have

$$
\left|D \psi_{\varepsilon}^{ \pm}\right|^{\frac{2 n}{n+1}}= \begin{cases}{\left[\frac{n}{\varepsilon} f\left(\frac{r}{\varepsilon}\right)^{\frac{n+1}{2}}\right]^{\frac{2 n}{n+1}}=n^{\frac{2 n}{n+1}} \varepsilon^{-\frac{2 n}{n+1}} f\left(\frac{r}{\varepsilon}\right)^{n}} & \text { if } r \leq \xi, \\ \left| \pm \varepsilon_{0} \nabla \eta \cdot \theta+f\left(\frac{\xi}{\varepsilon}\right)^{\frac{n}{2}} \nabla \eta \cdot \psi_{0}\right|^{\frac{2 n}{n+1}} & \text { if } \xi \leq r \leq 2 \xi \\ 0 & \text { if } r \geq 2 \xi .\end{cases}
$$

In the following, the notation $C$ will stand for positive constants (eventually depending on the dimension $n$, but not on $\varepsilon$ ) which can differ from one line to another. Equation (3.5) yields the following estimates:

$$
\int_{B(p, \xi)}\left|D \psi_{\varepsilon}^{ \pm}\right|^{\frac{2 n}{n+1}}=\varepsilon^{n-\frac{2 n}{n+1}} n^{\frac{2 n}{n+1}} \int_{B\left(p, \frac{\xi}{\varepsilon}\right)} f^{n} \leq \varepsilon^{n-\frac{2 n}{n+1}} n^{\frac{2 n}{n+1}} \int_{\mathbb{R}^{n}} f^{n}
$$


and

$$
\begin{aligned}
\int_{B(p, 2 \xi) \backslash B(p, \xi)}\left|D \psi_{\varepsilon}^{ \pm}\right|^{\frac{2 n}{n+1}} \leq & C \int_{B(p, 2 \xi) \backslash B(p, \xi)}\left|\varepsilon_{0} \nabla \eta \cdot \theta\right|^{\frac{2 n}{n+1}} \\
& +C^{\prime} \int_{B(p, 2 \xi) \backslash B(p, \xi)}\left|f\left(\frac{\xi}{\varepsilon}\right)^{\frac{n}{2}} \nabla \eta \cdot \psi_{0}\right|^{\frac{2 n}{n+1}} \\
\leq & C \varepsilon^{\frac{n(2 n-1)}{n+1}}+C \varepsilon^{\frac{n(2 n-1)}{n+1}} \leq C \varepsilon^{\frac{n(2 n-1)}{n+1}}
\end{aligned}
$$

since $\varepsilon_{0} \leq C \varepsilon^{n-1},|\nabla \eta| \leq \frac{2}{\xi}, \operatorname{Vol}(B(p, 2 \xi) \backslash B(p, \xi)) \leq C \xi^{n}$ and $|\theta| \leq C \xi$ on $B(p, 2 \xi)$, as well as $f\left(\frac{\xi}{\varepsilon}\right)^{\frac{n}{2}} \leq C \varepsilon^{\frac{n^{2}}{n+1}}$.

Therefore,

$\left(\int_{M}\left|D \psi_{\varepsilon}^{ \pm}\right|^{\frac{2 n}{n+1}}\right)^{\frac{n+1}{n}} \leq \varepsilon^{n-1} n^{2} I^{1+\frac{1}{n}}\left[1+C \varepsilon^{\frac{n^{2}}{n+1}}\right]=\varepsilon^{n-1} n^{2} I^{1+\frac{1}{n}}\left[1+o\left(\varepsilon^{n-1}\right)\right]$,

where $I:=\int_{\mathbb{R}^{n}} f^{n}$.

If we set

$$
\nu:=\left\langle\psi_{0}, \psi_{1}\right\rangle
$$

we have

$$
\begin{aligned}
\Re e & \left\langle D \psi_{\varepsilon}^{ \pm}, \psi_{\varepsilon}^{ \pm}\right\rangle_{\mid B(p, \xi)} \\
& =\Re e\left\langle \pm \frac{n}{\varepsilon} f\left(\frac{r}{\varepsilon}\right)^{\frac{n}{2}+1}\left(1 \mp \frac{x}{\varepsilon}\right) \cdot \psi_{0}, f\left(\frac{r}{\varepsilon}\right)^{\frac{n}{2}}\left(1 \mp \frac{x}{\varepsilon}\right) \cdot \psi_{0} \mp \varepsilon_{0} \psi_{1}\right\rangle \\
& = \pm \frac{n}{\varepsilon} f\left(\frac{r}{\varepsilon}\right)^{n}-\frac{n}{\varepsilon} \varepsilon_{0} f\left(\frac{r}{\varepsilon}\right)^{\frac{n}{2}+1} \Re e(\nu) \pm \frac{n}{\varepsilon} \varepsilon_{0} f\left(\frac{r}{\varepsilon}\right)^{\frac{n}{2}+1} \Re e\left\langle\frac{x}{\varepsilon} \cdot \psi_{0}, \psi_{1}\right\rangle
\end{aligned}
$$

and hence, since by symmetry the last term vanishes when integrating over $B(p, \xi)$, we have

$$
\int_{B(p, \xi)} \Re e\left\langle D \psi_{\varepsilon}^{ \pm}, \psi_{\varepsilon}^{ \pm}\right\rangle=n \varepsilon^{n-1}\left[ \pm \int_{B\left(p, \frac{\xi}{\varepsilon}\right)} f(r)^{n}-\Re e(\nu) \varepsilon_{0} \int_{B\left(p, \frac{\xi}{\varepsilon}\right)} f(r)^{\frac{n}{2}+1}\right] .
$$

Moreover,

$$
\int_{B\left(p, \frac{\xi}{\varepsilon}\right)} f(r)^{n}=I-\omega_{n-1} \int_{\frac{\xi}{\varepsilon}}^{+\infty} r^{n-1} f(r)^{n} \mathrm{~d} r
$$

Since

$$
\int_{\frac{\xi}{\varepsilon}}^{+\infty} r^{n-1} f(r)^{n} \mathrm{~d} r \leq \int_{\frac{\xi}{\varepsilon}}^{+\infty} r^{-(n+1)} \mathrm{d} r \leq C \varepsilon^{\frac{n^{2}}{n+1}}
$$


and since $\varepsilon_{0} \sim \varepsilon^{n-1}$ when $\varepsilon \rightarrow 0$, we have, for $\Re e(\nu)<0$,

$$
\int_{B(p, \xi)} \Re e\left\langle D \psi_{\varepsilon}^{+}, \psi_{\varepsilon}^{+}\right\rangle \geq n \varepsilon^{n-1}\left[I-C_{0} \Re e(\nu) \varepsilon^{n-1}+o\left(\varepsilon^{n-1}\right)\right],
$$

and for $\Re e(\nu)>0$,

$$
-\int_{B(p, \xi)} \Re e\left\langle D \psi_{\varepsilon}^{-}, \psi_{\varepsilon}^{-}\right\rangle \geq n \varepsilon^{n-1}\left[I+C_{0} \Re e(\nu) \varepsilon^{n-1}+o\left(\varepsilon^{n-1}\right)\right],
$$

where

$$
C_{0}=\int_{\mathbb{R}^{n}} f(r)^{\frac{n}{2}+1}
$$

We also have

$$
\begin{aligned}
& \Re e\left\langle D \psi_{\varepsilon}^{ \pm}, \psi_{\varepsilon}^{ \pm}\right\rangle_{\mid B(p, 2 \xi) \backslash B(p, \xi)} \\
& \quad=\Re e\left\langle \pm \varepsilon_{0} \nabla \eta \cdot \theta+f\left(\frac{\xi}{\varepsilon}\right)^{\frac{n}{2}} \nabla \eta \cdot \psi_{0}, \mp \varepsilon_{0}(\psi-\eta \theta)+\eta f\left(\frac{\xi}{\varepsilon}\right)^{\frac{n}{2}} \psi_{0}\right\rangle \\
& \quad=\Re e\left\langle \pm \varepsilon_{0} \nabla \eta \cdot \theta+f\left(\frac{\xi}{\varepsilon}\right)^{\frac{n}{2}} \nabla \eta \cdot \psi_{0}, \mp \varepsilon_{0} \psi\right\rangle
\end{aligned}
$$

since $\Re e\langle\nabla \eta \cdot \theta, \theta\rangle=0, \Re e\left\langle\nabla \eta \cdot \psi_{0}, \psi_{0}\right\rangle=0$ and

$$
\Re e\left\langle\nabla \eta \cdot \psi_{0}, \theta\right\rangle+\Re e\left\langle\nabla \eta \cdot \theta, \psi_{0}\right\rangle=\Re e\left\langle\nabla \eta \cdot \psi_{0}, \theta\right\rangle-\Re e \overline{\left\langle\nabla \eta \cdot \psi_{0}, \theta\right\rangle}=0 .
$$

Now, we write that on $B(p, 2 \xi) \backslash B(p, \xi), \varepsilon_{0}|\psi| \leq C \varepsilon^{n-1} \xi^{-(n-1)}, \varepsilon_{0}|\nabla \eta||\theta| \leq$ $C \varepsilon^{n-1}$ and $f\left(\frac{\xi}{\varepsilon}\right)^{\frac{n}{2}}|\nabla \eta| \leq C \varepsilon^{n-1}$. This leads to

$$
\Re e\left\langle D \psi_{\varepsilon}^{ \pm}, \psi_{\varepsilon}^{ \pm}\right\rangle_{\mid B(p, 2 \xi) \backslash B(p, \xi)} \leq C \varepsilon^{2 n-2} \xi^{1-n},
$$

which yields

$$
\int_{B(p, 2 \xi) \backslash B(p, \xi)} \Re e\left\langle D \psi_{\varepsilon}^{ \pm}, \psi_{\varepsilon}^{ \pm}\right\rangle=O\left(\varepsilon^{2 n-2+\frac{1}{n+1}}\right)=o\left(\varepsilon^{2 n-2}\right) .
$$

Therefore, since

$$
\Re e\left\langle D \psi_{\varepsilon}^{ \pm}, \psi_{\varepsilon}^{ \pm}\right\rangle_{\mid M \backslash B(p, 2 \xi)}=0,
$$

from Equations (3.7), (3.8), (3.9), we have for $\Re e(\nu)<0$,

$$
\int_{M} \Re e\left\langle D \psi_{\varepsilon}^{+}, \psi_{\varepsilon}^{+}\right\rangle \geq n \varepsilon^{n-1} I\left[1-C_{0} \Re e(\nu) \varepsilon^{n-1}+o\left(\varepsilon^{n-1}\right)\right],
$$


and for $\Re e(\nu)>0$,

$$
-\int_{M} \Re e\left\langle D \psi_{\varepsilon}^{-}, \psi_{\varepsilon}^{-}\right\rangle \geq n \varepsilon^{n-1} I\left[1+C_{0} \Re e(\nu) \varepsilon^{n-1}+o\left(\varepsilon^{n-1}\right)\right],
$$

Together with (3.6), we then have, for $\Re e(\nu)<0$,

$$
J\left(\psi_{\varepsilon}^{+}\right) \leq n I^{\frac{1}{n}} \frac{1+o\left(\varepsilon^{n-1}\right)}{1-C_{0} \Re e(\nu) \varepsilon^{n-1}+o\left(\varepsilon^{n-1}\right)},
$$

and for $\Re e(\nu)>0$,

$$
-J\left(\psi_{\varepsilon}^{-}\right) \leq n I^{\frac{1}{n}} \frac{1+o\left(\varepsilon^{n-1}\right)}{1+C_{0} \Re e(\nu) \varepsilon^{n-1}+o\left(\varepsilon^{n-1}\right)} .
$$

For $\varepsilon$ small enough, we obtain

$$
J\left(\psi_{\varepsilon}^{+}\right)<n I^{\frac{1}{n}} \text { and }-J\left(\psi_{\varepsilon}^{-}\right)<n I^{\frac{1}{n}}
$$

Recall now the following fact: let $p$ be any point of the round sphere $\mathbb{S}^{n}$. Then, $\mathbb{S}^{n} \backslash\{p\}$ is isometric to $\mathbb{R}^{n}$ with the metric

$$
g_{\mathbb{S}}=4 f^{2} g_{\text {eucl }} \text {. }
$$

Therefore,

$$
2^{n} I=\int_{\mathbb{R}^{n}} 2^{n} f^{n}=\omega_{n}
$$

which yields, for $\Re e(\nu)<0$ (resp. for $\Re e(\nu)>0$ )

$$
J\left(\psi_{\varepsilon}^{+}\right)<\frac{n}{2} \omega_{n}^{\frac{1}{n}} \quad\left(\text { resp. } \quad-J\left(\psi_{\varepsilon}^{-}\right)<\frac{n}{2} \omega_{n}^{\frac{1}{n}}\right) .
$$

Hence, by (3.1) and (3.2), the proof of Theorem 3.1 is now complete.

\section{Proofs of Theorems 1.2 and 1.3.}

Let $p \in M$. Up to a conformal change of metric, we may assume that $g$ is flat near $p$. Assume that the mass endomorphism $\alpha_{p}$ possesses a non-zero eigenvalue $\nu$. Let $\psi_{0} \in \Sigma_{p}(M)$ be an eigenvector associated to $\nu$. Then, we set

$$
\psi=-\omega_{n-1} G_{D}(x, p) \psi_{0}
$$

The spinor field $\psi$ then satisfies the assumptions of Theorem 3.1 with $\psi_{1}=\nu \psi_{0}$. Theorem 3.1 implies that if $\nu>0$ then $\lambda_{\min }^{+}(M, g, \theta)<\frac{n}{2} \omega_{n}^{\frac{1}{n}}$ and if $\nu<0, \lambda_{\min }^{-}(M, g, \theta)<\frac{n}{2} \omega_{n}^{\frac{1}{n}}$. This proves Theorem 1.2. Now, if $n \not \equiv 3$ $\bmod 4$, the spectrum of the mass endomorphism is symmetric and hence if $\alpha_{p} \neq 0, \nu$ can be chosen positive or negative. This proves Theorem 1.3. 


\section{References.}

[1] R. L. Arnowitt, S. Deser, and C. W. Misner, Canonical analysis of general relativity, Recent developments in general relativity, Pergamon, Oxford, 1962, pp. 127-136.

[2] B. Ammann, A spin-conformal lower bound of the first positive Dirac eigenvalue, Diff. Geom. Appl. 18 (2003), 21-32.

[3] B. Ammann, The smallest Dirac eigenvalue in a spin-conformal class and cmc-immersions, Preprint, ArXiv math.DG/0309061

[4] B. Ammann, A variational problem in conformal spin Geometry, Habilitationsschrift, Universität Hamburg, May 2003.

[5] B. Ammann, E. Humbert, Positive mass theorem for the Yamabe problem on spin manifolds. Preprint

[6] M. F. Atiyah, V. K. Patodi, and I. M. Singer, Spectral asymmetry and Riemannian geometry. III, Math. Proc. Cambridge Philos. Soc. 79 (1976), no. 1, 71-99.

[7] T. Aubin, Équations différentielles non linéaires et problème de Yamabe concernant la courbure scalaire. J. Math. Pur. Appl., IX. Ser., 55:269296, 1976.

[8] C. Bär, Lower eigenvalue estimates for Dirac operators. Math. Ann., 293, 1992.

[9] M. Dahl, Prescribing eigenvalues of the Dirac operator, Preprint, ArXiv math.DG/0311172

[10] T. Friedrich, Dirac operators in Riemannian geometry, Graduate Studies in Mathematics, vol. 25, American Mathematical Society, Providence, RI, 2000.

[11] E. Hebey, Introduction à l'analyse non-linéaire sur les variétés, Diderot Éditeur, Arts et sciences, 1997.

[12] O. Hijazi, A conformal lower bound for the smallest eigenvalue of the Dirac operator and Killing Spinors, Comm. Math. Phys., 104 151-162, 1986 
[13] O. Hijazi, Première valeur propre de l'opérateur de Dirac et nombre de Yamabe, C. R. Acad. Sci. Paris, Série I 313, (1991), 865-868.

[14] N. Hitchin, Harmonic spinors, Adv. Math. 14 (1974), 1-55.

[15] H.-B. Lawson, M.-L. Michelsohn, Spin Geometry, Princeton University Press, Princeton 1989.

[16] J. M. Lee and T. H. Parker. The Yamabe problem. Bull. Am. Math. Soc., New Ser., 17 37-91, 1987.

[17] J. Lott, Eigenvalue bounds for the Dirac operator. Pacific J. of Math., 125, 117-126, 1986.

[18] R. Schoen. Conformal deformation of a Riemannian metric to constant scalar curvature. J. Diff. Geom., 20, 479-495, 1984.

[19] N.S. Trudinger. Remarks concerning the conformal deformation of Riemannian structures on compact manifolds. Ann. Sc. Norm. Super. Pisa, Sci. Fis. Mat., III. Ser., 22, 265-274, 1968.

[20] H. Yamabe. On a deformation of Riemannian structures on compact manifolds. Osaka Math. J., 12, 21-37, 1960.

Institut Élie CARTAN BP 239

UNIVERSITÉ DE NANCY 1

54506 VANDOeUvre-LÈs -NANCY CEDEX

FRANCE

E-mail address: ammann@iecn.u-nancy.fr

E-mail address: humbert@iecn.u-nancy.fr

E-mail address: morel@iecn.u-nancy.fr 